\title{
Acknowledgement to Reviewers of Journal of Fungi in 2019
}

Journal of Fungi Editorial Office

MDPI, St. Alban-Anlage 66, 4052 Basel, Switzerland

Published: 21 January 2020

The editorial team greatly appreciates the reviewers who have dedicated their considerable time and expertise to the journal's rigorous editorial process over the past 12 months, regardless of whether the papers are finally published or not. In 2019, a total of 116 papers were published in the journal, with a median time to first decision of 38 days and a median time from submission to publication of 18 days. The editors would like to express their sincere gratitude to the following reviewers for their generous contribution in 2019:

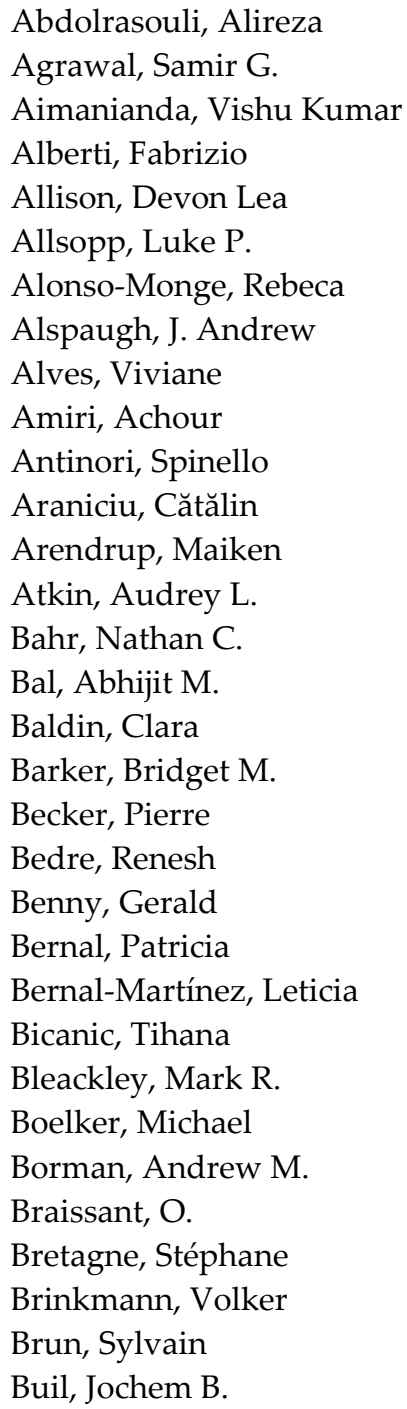

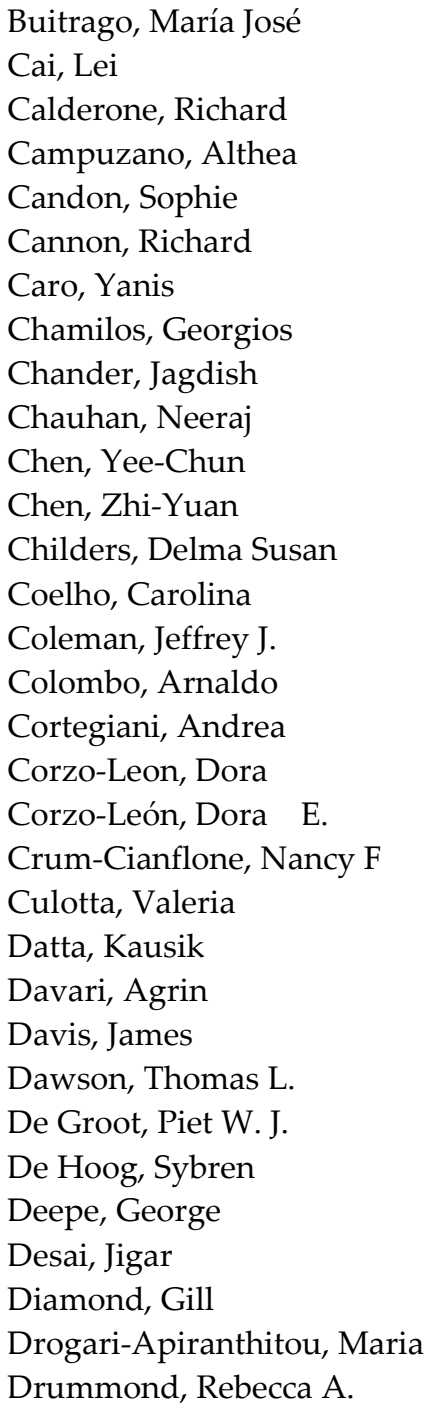


Dufossé, Laurent

Dymarska, Monika

Edgerton, Mira

Elad, Daniel

Erdman, Scott

Esher, Shannon

Espinel-Ingroff, Ana

Franco, Chris

Frealle, Emilie

Fuchs, Beth

Gago, Sara

Gambino, Alessio

Gandía, Mónica

Garcia-Diaz, Julia

Garcia-Sherman, Melissa

Garre, Victoriano

Gelli, Angie

Gonzalez, Gabriel

Grabinska, Kariona

Groll, Andreas

Guitard, Juliette

H. Caceres, Diego

Hage, Chadi

Hagen, Ferry

Halliday, Catriona

Hamill, Richard

Haneke, Eckart

Hao, Guixia

Harro, Janette

Hay, Rod

Hendrickx, Marijke

Hennequin, Christophe

Hennicke, Florian

Hillmann, Falk

Hoenigl, Martin

Hong, Chang Pyo

Ikeda, Kenichi

Jackson, Brendan R

Jacobsen, Ilse

Johnson, Elizabeth

Johnston, Simon

Junqueira, Juliana Campos

Kasanen, Risto

Kauffman, Carol

Kavanagh, Kevin

Kean, Ryan

Kidd, Sarah E.

Kirkland, Theo N.

Kirschner, Roland

Kitagaki, Hiroshi

Kong, Won-Sik

Kordalewska, Milena
Kovacs, Renato

Krom, Bastian

Kubicek, Christian

Kucharíková, Soňa

Kumar, Pradeep

Kumar, Rohitashw

Kumaresan, Pappanaicken

Lachance, Marc-André

Lackner, Michaela

Lake, Douglas F.

Lanternier, Fanny

Larriba, German

Lavergne, Rose-Anne

Lee, Chao-Hung

Lee, Soo Chan

Legrand, Mélanie

Leitão, Ana

Levesque, Eric

Li, Shaojie

Li, TaoTao

Li, Xiu-Mei

Lin, Chi-Jan

Lindsley, Mark D.

Litvintseva, Anastasia P.

Lombardi, Lisa

Lopez-Ribot, Jose

Lorenz, Alexander

Lundy, Fionnuala T

Lutterloh, Emily

Lyman, Meghan

Maddi, Abhiram

Malacrinò, Antonino

Mantadakis, Elpis

Markinson, Bryan

Marques, Claudia

Martinez, Luis

McClelland, Erin E.

Meya, David B.

Millon, Laurence

Mora-Montes, Hector

Moran, Gary

Morio, Florent

Mourad, Ahmad

Mouyna, Isabelle

Mukaremera, Liliane

Munoz, Jose

Nacher, Mathieu

Nakayama, Haruo

Nanjappa, Som G.

Narra, Hema Prasad

Nobbs, Angela

Nosanchuk, Joshua 
Nowrousian, Minou

Oniga, Smaranda Dafina

Overy, David P.

Özenci, Volkan

Pagano, Marcela

Park, Young-Jin

Pasqualoto, Alessandro C.

Pawłowska, Julia

Pedrosa, Ana Filipa

Perochon, Alexandre

Peters, Brandilyn

Peters, Brian

Petraitis, Vidmantas

Pfaller, Michael A.

Pires, Regina Helena

Ponce De León, Alfredo

Ponikau, Jens U.

Popolo, Laura Maria

Portilla, Maribel

Posch, Wilfried

Prigione, Valeria

Queiroz-Telles, Flavio

Ramachandiran, Iyappan

Rangel, Drauzio E.N.

Rapala-Kozik, Maria

Rappleye, Chad

Reed, Kevin F.M.

Richardson, Riina

Robert-Gangneux, Florence

Robinson, Seri

Romo, Jesus

Rostislav, Zemek

Rotstein, Coleman

Rupp, Steffen

Sabino, Raquel

Samaranayake, Lakshman

Sampaio, Paula

Schmidt, Martin

Schöps, Ricardo

Schwarz, Patrick

Segal, Esther

Sellam, Adnane

Sexton, Joseph D.

Sharma, Cheshta

Sharp, Jo

Shaw, Brian D.

Shi, Meiqing

Shoham, Shmuel

Silva, Sónia Carina

Simonetti, Giovanna

Sipsas, Nikolaos V.

Sobrado, Pablo
Solano, Francisco

Staniszewska, Monika

Stchigel, Alberto Miguel

Stevens, David

Sun, Man-Hong

Swidergall, Marc

Talbot, Nick

Taylor-Smith, Leanne

Teixeira, Marcus M

Thekkiniath, Jose

Thielen, Beth

Torović, Ljilja

Torren, Laura Vilanova

Uzman, Deniz

Valdes, Audrey

Vallabhaneni, Snigdha

Vargas, Sergio

Vautier, Simon

Vlahovic, Tracey

Voss, Andreas

Wang, Zhe

Warris, Adilia

Welsh, Rory

Wengenack, Nancy

White, Lewis

Wickes, Brian L.

Williamson, Peter R.

Wimmer, Reinhard

Wöstemeyer, Johannes

Wurster, Sebastian

$\mathrm{Xu}$, Jianping

$\mathrm{Xu}$, Jintao

Xue, Chaoyang

Yan, Dong-Hui

Yun, Yingzi

Zaias, Nardo

Zhao, Yanan 
(C) 2020 by the author. Licensee MDPI, Basel, Switzerland. This article is an open access article distributed under the terms and conditions of the Creative Commons Attribution (CC BY) license (http://creativecommons.org/licenses/by/4.0/). 